Document downloaded from:

http://hdl.handle.net/10251/105343

This paper must be cited as:

Torrijo, F.; Garzón-Roca, J.; Company Rodríguez, J.; Cobos Campos, G. (2018). Estimation of cerchar abrasivity index of andesitic rocks in Ecuador from chemical compounds and petrographical properties using regression analyses. Bulletin of Engineering Geology and the Environment. 1-14. doi:10.1007/s10064-018-1306-6

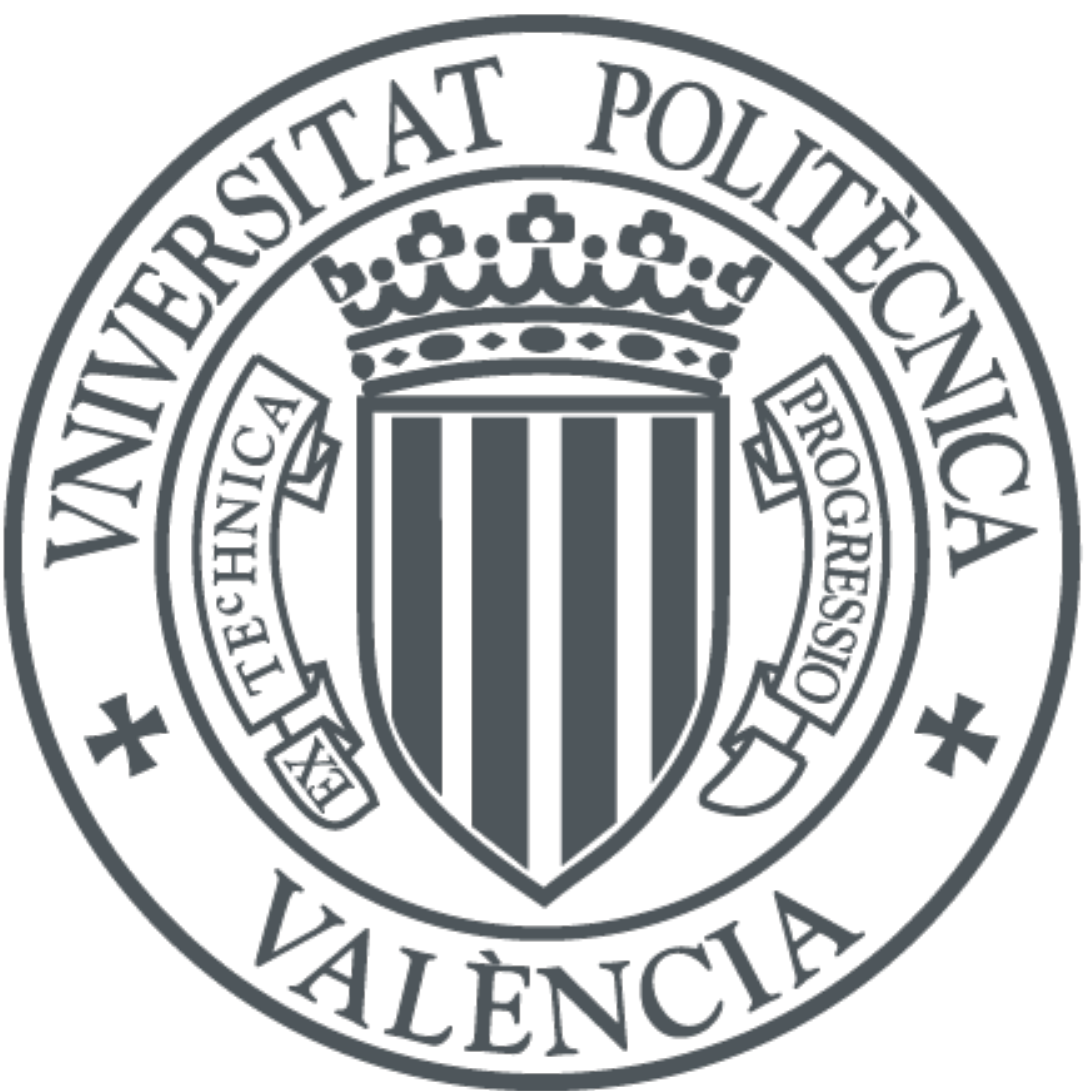

The final publication is available at

https://doi.org/10.1007/s10064-018-1306-6

Copyright Springer-Verlag

Additional Information 


\section{ESTIMATION OF CERCHAR ABRASIVITY INDEX OF ANDESITIC ROCKS IN ECUADOR FROM CHEMICAL COMPOUNDS AND PETROGRAPHICAL PROPERTIES USING REGRESSION ANALYSES}

F. Javier Torrijo ${ }^{\mathrm{a}, *}$, Julio Garzón-Roca ${ }^{\mathrm{a}}$, Julio Company ${ }^{\mathrm{a}}$, Guillermo Cobos $^{\mathrm{a}}$

${ }^{a}$ Department of Geotechnical Engineering, Universitat Politècnica de València, Camino de Vera s/n, 46022, Valencia, Spain.

${ }^{*}$ Corresponding author. Tel.: +34 963877 582; fax: +34 963877 569; E-mail address: fratorec@ @trr.upv.es

\section{Abstract}

An important issue in any rock engineering project is the adequate prediction of tool consumption. Excavation tools are subjected to wear and repair/replacement of those tools is usually an important expense on any excavation budget. The key factor that affects wear of excavation tools is rock abrasivity. In mining and civil engineering, rock abrasivity is typically measured by the Cerchar Abrasivity Index $(C A I)$, which is obtained in laboratory from a Cerchar abrasivity test. This paper studied the relation between $C A I$ and the chemical compounds and petrographical properties of andesitic rocks coming from the central area of Ecuador. A series of regression analyses are performed to study the influence of the different chemical compounds and petrographical properties on the $C A I$ value. Results show that it is possible to make a good estimation of $C A I$ from the plagioclase grain size and/or the content in $\mathrm{SiO}_{2}, \mathrm{FeO}, \mathrm{MgO}, \mathrm{CaO}, \mathrm{Na}_{2} \mathrm{O}$ and $\mathrm{K}_{2} \mathrm{O}$ compounds.

Keywords: Cerchar Abrasivity Index; andesitic rock; chemical compounds; petrographical properties. 


\section{Introduction}

Material tool consumption is one of the main indicators of rock excavation in mining and civil engineering projects (e.g. tunnelling, underground mining and quarrying). In fact, an important issue in any rock project is the adequate prediction of tool consumption, being especially during the tendering stage a significant factor in the estimation of expenses. Either rock excavation is performed by conventional drilling and blasting or by means of mechanized excavators such as TBMs, roadheaders and dozers, excavation tools are exposed to wearing. Although wear partially depends on the machinery being used for excavation and the geological conditions, the key factor that affects wear of excavation (cutting) tools is rock abrasivity. Repair and replacement of rock cutting tools as well as other machine components in contact with the rock during excavation (which are also subjected to wear) have been reported to be an important amount on any excavation budget (Fowell and Abu Baker, 2007; Hamzaban et al., 2014b). Hence rock abrasivity is a very important factor to consider in the operating costs and performance of any mechanical rock excavation work. It should also be noted that mechanical rock excavation is usually carried out by machines of high cost and in most cases site specific, thus selecting the adequate cutter tool according to the rock abrasivity to be excavated is essential when looking for an optimum performance.

The abrasivity of rocks can be related to their petrographic composition, especially with the amount of hard minerals like quartz (Käsling and Tara, 2010), but other features such as the mean grain size, type of cement, and degree of cementation can influence the abrasivity of a rock (West, 1989; Yarali et al., 2008). Petrological methods may be used to estimate abrasivity (West, 1989). That includes Mohs's scratch hardness, Vickers hardness, silica content or microscopic examination of a thin section. Mechanical parameters such as uniaxial compression strength, tensile strength and fracture toughness may also be taken into account (Alber, 2008; Deliormanl1, 2012). However, typically abrasivity of rocks is more technically obtained from laboratory tests and associated with some kind of model or an index. Nowadays, Cerchar abrasivity test (CERCHAR, 1986; ASTM- 
D7625, 2010; Alber et al., 2014) is probably the most common tests used to evaluate abrasivity of rocks, especially in the area of civil engineering (tunnelling), thanks to its simplicity and dependable results (Atkinson et al., 1986a, 1986b). From this test, the Cerchar Abrasivity Index $(C A I)$ is obtained and used as the parameter which describes the abrasivity of rocks. Both the test and how to obtain that index will be explained later in this paper.

Several researches studied the dominant factors of CAI (Rostami et al., 2014) and the effects that different aspects can have on $C A I$, such as quartz content, grain size and matrix properties (Suana and Peters, 1982; Lassnig et al., 2008), rock strength (Al-Ameen and Wallner, 1994) stress dependency (Alber, 2007) or testing conditions, procedures and materials used to conduct the test (Al-Ameen and Wallner, 1994; Plinninger et al., 2003; Michalakopoulos et al., 2005; Fowell and Abu Bakar, 2007; Lassnig et al., 2008; Hamzaban et al., 2014a; Rostami et al., 2014). Likewise, some investigations analysed and correlate $C A I$ with mechanical and/or geological properties, including chemical compounds, petrographical properties, equivalent quartz content, uniaxial compression strength or Young modulus (Plinninger et al., 2003; Kahraman et al., 2010; Deliormanl1, 2011; Moradizadeh et al., 2013; Er and Tugrul, 2016a, 2016b; Majeed and Bakar, 2016). Nevertheless, most of the mentioned studies only dealt with some rock specific samples (e.g. granitic rocks), and up to now there is not a clear evidence that their results may be completely extrapolated and used when facing other rock formations.

In this paper a relation between $C A I$ and the chemical compounds and petrographical properties of andesitic rocks is investigated. A total of 73 andesitic samples coming from the central area of Ecuador are subjecting to Cerchar abrasivity tests and are chemically and petrographically analysed, in order to establish both $C A I$ and their chemical and modal compounds as well as minerals grain size. A series of regression analyses are performed studying the influence of the different chemical compounds and petrographical properties on $C A I$ value. Regression analyses are frequently used in engineering and have recently demonstrated to be effective to correlate $C A I$ with mechanical and 
geological properties (Er and Tugrul, 2016a, 2016b; Majeed and Bakar, 2016). Both simple regression and multiregression models are considered in this paper.

\section{Geographical Setting and Geological Framework}

The andesite samples analysed in this study come from the Bombolí area (Mejía canton, Pichincha province, Ecuador), where the construction of a new road tunnel is currently being built. The ca. 2 kilometer-long Bombolí tunnel runs between the kilometric points (kp) 20+221 and 21+959 of the E-20 road Alóag-Santo Domingo, approximately $50 \mathrm{Km}$ South-West of the city of Quito (Fig. 1).

The road Alóag-Santo Domingo stretches over mafic lavas and volcano-sedimentary rocks of the Western Cordillera of Ecuador, a north-south trending chain which is one of the two major branches of the Ecuadorian Andean Mountain Range (Vallejo, 2007; Vallejo et al., 2009; Vera, 2016).

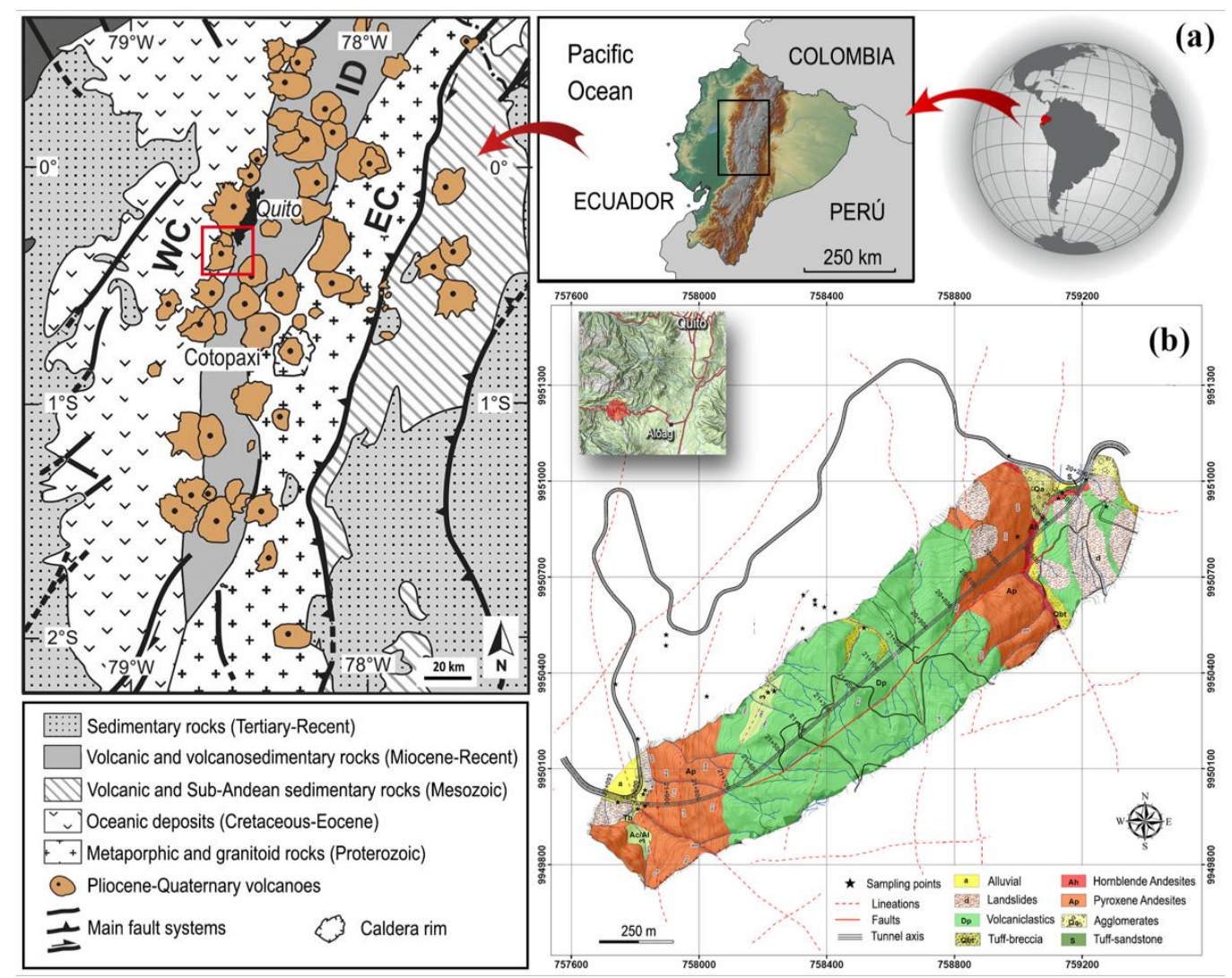

Fig. 1. Sketch maps showing the location of the studied area. The successive insets show the position of the detailed maps: a) Geological simplified map of the Ecuadorian Andes showing the main stratigraphic units outcropping in the region. (Modified from Vezzoli et al. 2017). Abbreviations: EC = Eastern Cordillera; ID = Interandean Depression; WC $=$ Western Cordillera; b) Detailed map of the Bomboli road tunnel showing the different volcano-sedimentary materials affected by mechanical rock excavations and sample locations. 
In the project area, the extensive exposures of volcano-sedimentary materials can be mainly referred to the Silante Formation, an upper Maastrichtian-Paleocene volcanic unit whose type section crops out along the Alóag-Santo Domingo road. The Silante Formation consists (Fig. 2) of a thick succession of andesitic volcaniclastic deposits (fluvial conglomerates and Breccias, mudstones, siltstones and tuffaceous sandstones) with intercalations of andesites, dacites and breccias (Boland et al., 2000).

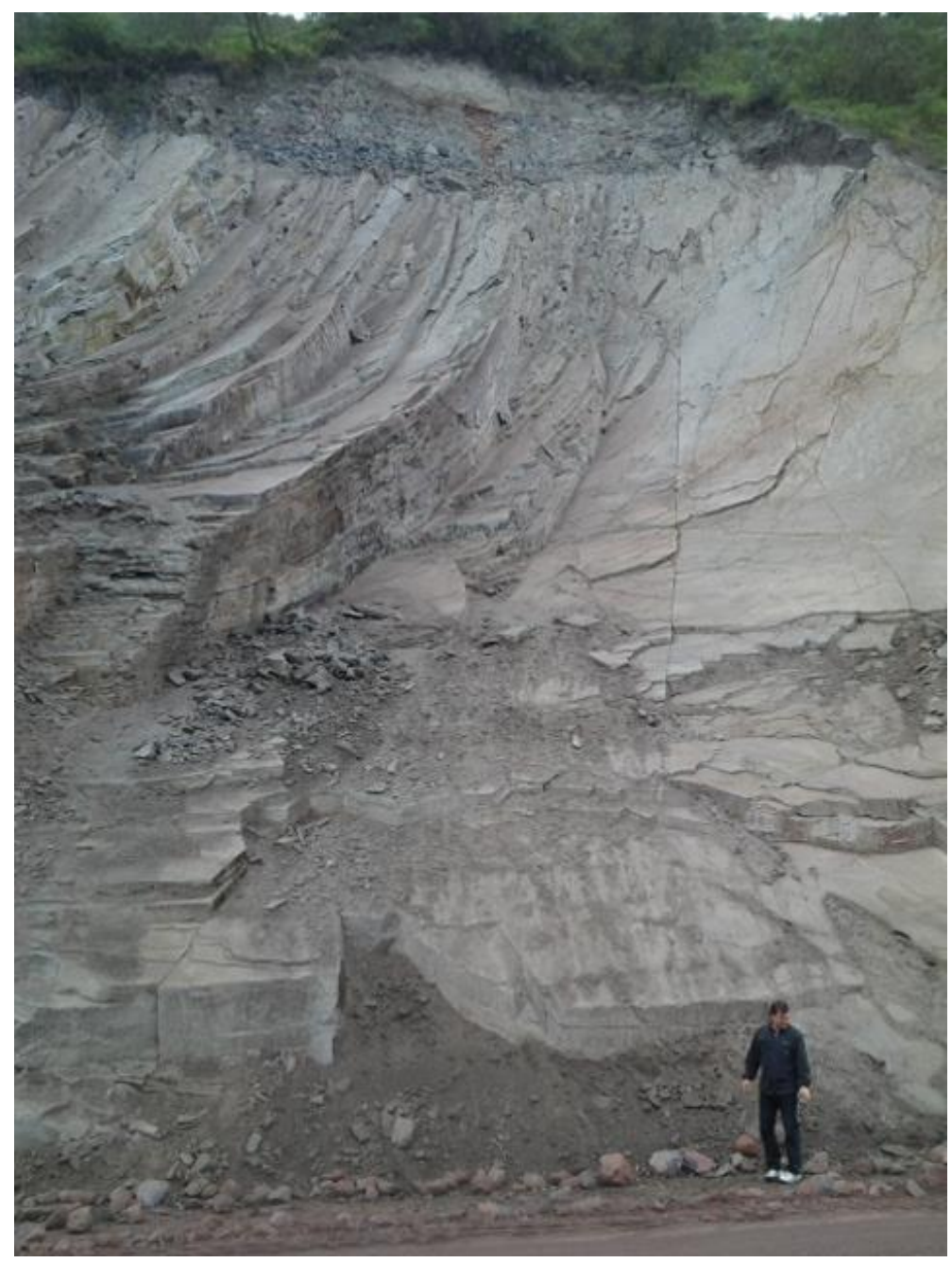

Fig. 2. View of the andesitic rocks studied in the project area.

\section{Experimental study}

103 Up to 73 andesitic rock samples from the project area were selected for mechanical and

104 petrographic analyses. Samples were extracted from three locations along the Bombolí area: 27

105 samples belonged to inside of tunnel, and will refer thereafter as TB; 16 samples were obtained 
106 from the slopes, TT samples thereafter; and 30 samples corresponded to the road, refer as VC

107 thereafter.

108 3.1. XRF analysis

109 The main chemical compounds of the andesitic samples were identified by a semi-quantitative

110 chemical analysis with X-ray fluorescence spectroscopy, carried out using a Perkin-Elmer 3030.

111 3.2. Petrographical characteristics

112 Thin sections were prepared for the 73 andesitic rock samples and studied under a petrographic

113 polarizing microscope for determining grain size and quantitative mineral content.

114 3.3. Physical and mechanical properties and Cerchar abrasivity index

115 Several tests were conducted on each group of samples to set their physical and mechanical

116 properties. Those tests included determining the unit weight and obtaining the uniaxial compression

117 strength and the tensile strength of the intact rock. Tests were conducted based on ISRM (2007) and

118 ASTM standards (ASTM D7012; ASTM D3967).

119 The 73 andesitic rock samples were subjected to Cerchar abrasivity tests. This test, introduced in the 1970s by the Centre d'Etudes et Recherches des Charbonages de France (CERCHAR) for assessing abrasivity in the coal mining industry (Yarali et al., 2008; Kasling and Thuro, 2010), was later adopted by the tunnelling industry (West, 1989; Rostami et al., 2014), and is nowadays typically selected as a tool to quantify rock abrasivity in predicting tool wear during hard rock tunnelling. Cerchar abrasivity test (Fig. 3) measures the wear on the tip of a steel stylus having a Rockwell Hardness of HRC 55. Two standards exist for this test method: the French standard NF P 94-430-1 (2000) and ASTM D7625-10 (2010). The test presented in this paper followed the former and ISRM suggested method (Alber et al., 2014), as well as the original specifications of the test

128 (CERCHAR, 1986). To measure the wear flat of the Cerchar test stylus, side view method was used, since that introduces less statistically significant error in the measured values of $C A I$. The stylus scratches the surface of a rough rock sample over a distance of $10 \mathrm{~mm}$ under static load of 70 
131 N. The wear surface of the stylus tip is afterwards measured under a microscope to an accuracy of $1320.01 \mathrm{~mm}$. The wear surface, stated in units of $0.01 \mathrm{~mm}$, is then multiplied by 10 to obtain the 133 Cerchar Abrasivity Index (CAI), which is a dimensionless unit value. The test is performed at least 134 five times on the same rock surface by using a fresh re-sharpened stylus each time and then taking 135 the arithmetic mean of the measured values.

136

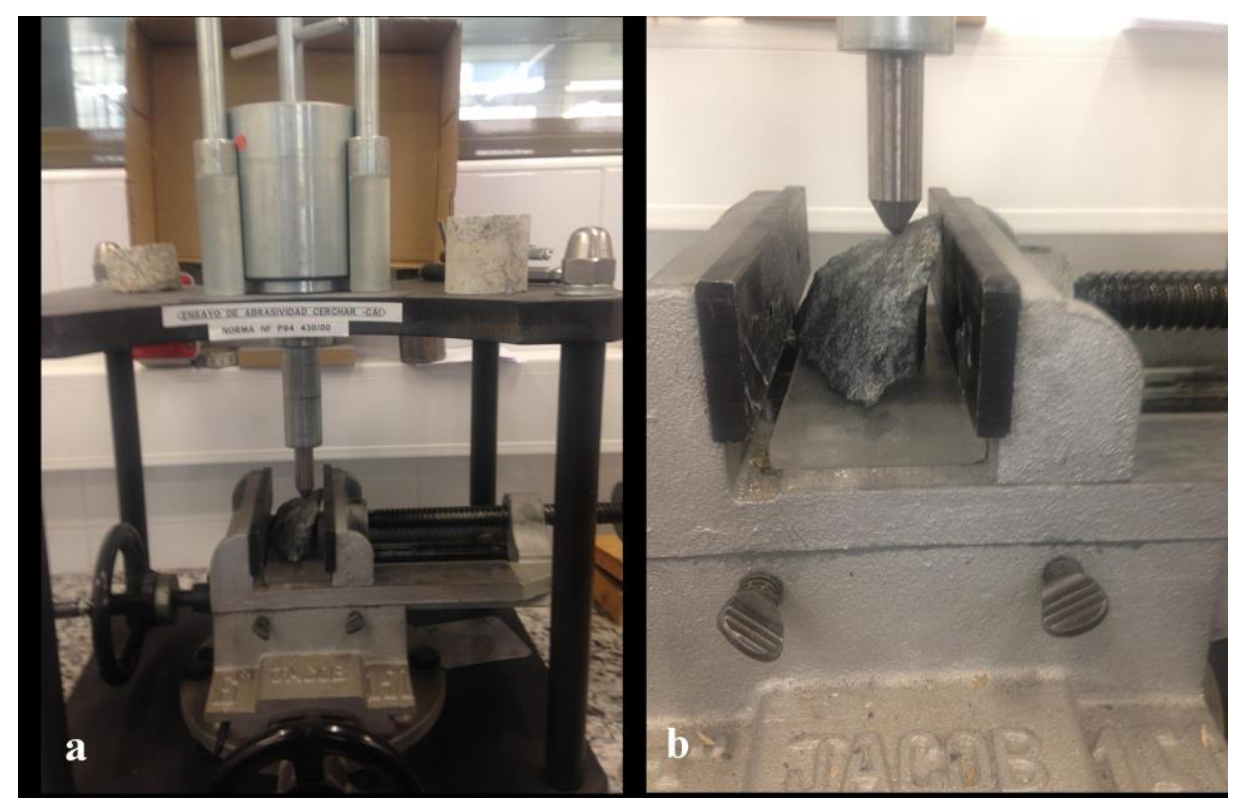

Fig. 3. Cerchar abrasivity test conducted: a) Cerchar device; b) Detail of the rock sample and the steel stylus.

The French standard AFNOR NF P 94-430-1 (2000) was follow. Tests were performed at the laboratories of the Department of Geotechnical Engineering of the Technical University of Valencia, by one technician. The tip of a steel stylus had a Rockwell Hardness of HRC 55.

\section{Results}

\subsection{XRF analysis}

The andesitic samples under study are mainly composed (Table 1) of $\mathrm{SiO}_{2}$, which represent ca. $50 \%$ or more in nearly all cases. The second more abundant compound is $\mathrm{Al}_{2} \mathrm{O}_{3}$, with ca. $15 \%$, followed by the $\mathrm{CaO}$, with ca. $10 \%$. Other compounds such as $\mathrm{Fe}_{2} \mathrm{O}_{3}, \mathrm{FeO}, \mathrm{MgO}, \mathrm{Na}_{2} \mathrm{O}, \mathrm{K}_{2} \mathrm{O}$ appear in small quantities (between $2 \%$ and $10 \%$ on average) and some traces of $\mathrm{TiO}_{2}, \mathrm{MnO}, \mathrm{P}_{2} \mathrm{O}_{5}$ are also found in the samples. 
Table 1. Chemical compounds of andesitic samples.

\begin{tabular}{|c|c|c|c|c|c|c|c|c|c|c|c|}
\hline \multirow{2}{*}{ Sample } & \multicolumn{11}{|c|}{ Chemical compounds (\%) } \\
\hline & $\mathrm{SiO}_{2}$ & $\mathrm{TiO}_{2}$ & $\mathrm{Al}_{2} \mathrm{O}_{3}$ & $\mathrm{Fe}_{2} \mathrm{O}_{3}$ & $\mathrm{FeO}$ & $\mathrm{MnO}$ & $\mathrm{MgO}$ & $\mathrm{CaO}$ & $\mathrm{Na}_{2} \mathrm{O}$ & $\mathrm{K}_{2} \mathrm{O}$ & $\mathrm{P}_{2} \mathrm{O}_{5}$ \\
\hline TB1 & 55.21 & 0.88 & 16.35 & 5.53 & 4.09 & 0.15 & 3.36 & 9.06 & 3.53 & 1.64 & 0.20 \\
\hline TB2 & 61.33 & 0.89 & 10.98 & 2.39 & 3.98 & 0.19 & 3.40 & 11.51 & 3.25 & 1.88 & 0.20 \\
\hline TB3 & 64.16 & 0.90 & 11.22 & 1.79 & 3.87 & 0.23 & 3.44 & 9.10 & 2.97 & 2.12 & 0.20 \\
\hline TB4 & 55.30 & 0.91 & 16.29 & 1.87 & 3.76 & 0.27 & 3.48 & 12.87 & 2.69 & 2.36 & 0.20 \\
\hline TB5 & 60.72 & 0.92 & 6.90 & 2.93 & 3.65 & 0.31 & 3.52 & 15.84 & 2.41 & 2.60 & 0.20 \\
\hline TB6 & 54.20 & 0.93 & 17.59 & 6.32 & 3.54 & 0.35 & 3.56 & 8.34 & 2.13 & 2.84 & 0.20 \\
\hline TB7 & 55.01 & 0.94 & 17.90 & 4.98 & 3.43 & 0.39 & 3.60 & 8.62 & 1.85 & 3.08 & 0.20 \\
\hline TB8 & 65.10 & 0.95 & 11.03 & 1.62 & 3.32 & 0.43 & 3.64 & 8.82 & 1.57 & 3.32 & 0.20 \\
\hline TB9 & 66.00 & 0.96 & 13.28 & 2.22 & 3.21 & 0.47 & 3.68 & 5.13 & 1.29 & 3.56 & 0.20 \\
\hline TB 10 & 60.76 & 0.60 & 13.81 & 4.06 & 8.27 & 0.90 & 2.31 & 4.14 & 3.53 & 1.42 & 0.19 \\
\hline TB11 & 58.91 & 1.03 & 15.38 & 1.82 & 2.46 & 0.34 & 2.54 & 12.00 & 3.25 & 2.07 & 0.19 \\
\hline TB12 & 59.16 & 0.58 & 14.86 & 3.73 & 8.65 & 1.10 & 2.20 & 4.43 & 2.99 & 2.12 & 0.19 \\
\hline TB13 & 56.30 & 0.57 & 16.64 & 5.19 & 8.84 & 1.25 & 2.15 & 5.41 & 2.69 & 0.76 & 0.19 \\
\hline TB14 & 57.15 & 0.56 & 15.76 & 4.19 & 3.03 & 1.41 & 2.09 & 8.71 & 2.52 & 4.39 & 0.19 \\
\hline TB15 & 55.90 & 0.54 & 17.20 & 4.11 & 8.15 & 1.42 & 2.04 & 5.06 & 2.42 & 2.96 & 0.19 \\
\hline TB16 & 58.12 & 0.53 & 12.79 & 4.69 & 9.41 & 1.73 & 1.98 & 3.81 & 1.85 & 5.00 & 0.08 \\
\hline TB17 & 53.31 & 0.52 & 17.13 & 4.52 & 9.60 & 1.26 & 1.93 & 8.73 & 1.57 & 1.24 & 0.19 \\
\hline TB18 & 54.70 & 0.51 & 14.55 & 4.45 & 4.79 & 2.04 & 1.87 & 14.93 & 1.29 & 0.67 & 0.19 \\
\hline TB19 & 45.92 & 0.48 & 18.43 & 5.86 & 9.98 & 2.20 & 1.82 & 8.27 & 3.53 & 3.32 & 0.19 \\
\hline TB20 & 53.08 & 0.48 & 9.63 & 4.56 & 10.17 & 2.35 & 1.77 & 11.02 & 3.25 & 3.56 & 0.13 \\
\hline TB21 & 62.43 & 0.83 & 17.62 & 5.78 & 6.86 & 0.35 & 1.71 & 0.11 & 2.69 & 1.42 & 0.19 \\
\hline TB22 & 56.54 & 0.46 & 16.15 & 3.94 & 10.55 & 0.39 & 1.66 & 5.65 & 2.41 & 2.07 & 0.19 \\
\hline TB23 & 50.31 & 0.45 & 17.15 & 4.49 & 10.74 & 0.43 & 1.60 & 10.39 & 2.13 & 2.12 & 0.19 \\
\hline TB24 & 45.92 & 0.43 & 19.37 & 5.86 & 5.35 & 0.47 & 1.55 & 18.24 & 1.85 & 0.76 & 0.19 \\
\hline TB25 & 75.00 & 0.42 & 2.98 & 1.55 & 1.27 & 0.90 & 1.49 & 10.23 & 1.57 & 4.39 & 0.19 \\
\hline TB26 & 61.62 & 0.41 & 14.61 & 3.01 & 1.09 & 0.94 & 1.44 & 10.19 & 3.53 & 2.96 & 0.19 \\
\hline TB27 & 53.31 & 0.40 & 16.29 & 4.52 & 0.57 & 2.04 & 1.39 & 16.17 & 3.25 & 1.88 & 0.19 \\
\hline TT1 & 51.46 & 0.39 & 14.32 & 4.86 & 11.69 & 2.20 & 1.33 & 8.75 & 2.69 & 2.12 & 0.19 \\
\hline TT2 & 53.77 & 1.16 & 16.05 & 5.40 & 2.77 & 2.35 & 4.48 & 10.65 & 2.41 & 0.76 & 0.20 \\
\hline TT3 & 59.08 & 1.17 & 15.38 & 3.48 & 1.31 & 0.35 & 4.52 & 8.00 & 2.13 & 4.39 & 0.20 \\
\hline TT4 & 55.85 & 1.18 & 16.35 & 5.02 & 2.31 & 0.39 & 4.56 & 10.73 & 0.45 & 2.96 & 0.20 \\
\hline TT5 & 60.23 & 1.19 & 15.03 & 3.27 & 0.68 & 0.43 & 4.60 & 9.48 & 0.17 & 4.80 & 0.12 \\
\hline TT6 & 54.46 & 1.20 & 16.78 & 4.31 & 0.57 & 1.43 & 4.64 & 11.45 & 3.53 & 1.42 & 0.20 \\
\hline TT7 & 69.46 & 1.21 & 9.90 & 1.90 & 0.46 & 0.03 & 4.65 & 6.87 & 3.25 & 2.07 & 0.20 \\
\hline TT8 & 43.38 & 1.22 & 20.14 & 7.29 & 0.35 & 1.51 & 4.72 & 16.10 & 2.97 & 2.12 & 0.20 \\
\hline TT9 & 60.23 & 0.97 & 15.02 & 3.15 & 4.15 & 0.51 & 3.72 & 8.29 & 3.00 & 0.76 & 0.20 \\
\hline TT10 & 53.08 & 0.98 & 17.20 & 4.56 & 2.99 & 0.55 & 3.12 & 9.73 & 3.20 & 4.39 & 0.20 \\
\hline TT11 & 55.85 & 0.99 & 16.36 & 5.02 & 2.05 & 0.59 & 3.80 & 9.58 & 2.60 & 2.96 & 0.20 \\
\hline TT12 & 57.92 & 1.00 & 14.79 & 3.68 & 4.81 & 0.63 & 3.84 & 11.08 & 0.17 & 1.88 & 0.20 \\
\hline TT13 & 53.54 & 0.90 & 17.06 & 4.48 & 4.09 & 0.15 & 3.36 & 10.58 & 3.53 & 2.12 & 0.19 \\
\hline TT14 & 54.46 & 0.81 & 16.78 & 5.28 & 3.98 & 0.19 & 3.40 & 9.77 & 3.25 & 1.98 & 0.10 \\
\hline TT15 & 54.92 & 0.90 & 16.64 & 4.23 & 3.87 & 0.23 & 3.44 & 10.48 & 2.97 & 2.12 & 0.20 \\
\hline TT16 & 52.15 & 1.01 & 16.62 & 4.73 & 5.66 & 0.67 & 3.88 & 11.42 & 3.00 & 0.76 & 0.09 \\
\hline VC1 & 50.54 & 1.05 & 17.97 & 4.45 & 2.22 & 0.83 & 4.04 & 11.21 & 3.20 & 4.39 & 0.10 \\
\hline VC2 & 51.00 & 0.86 & 14.44 & 4.94 & 2.11 & 0.87 & 4.08 & 15.95 & 2.60 & 2.96 & 0.19 \\
\hline VC3 & 56.77 & 1.02 & 16.08 & 2.30 & 2.55 & 0.71 & 3.92 & 8.34 & 2.99 & 5.00 & 0.32 \\
\hline VC4 & 53.54 & 1.03 & 17.05 & 4.48 & 2.50 & 0.75 & 3.96 & 11.98 & 3.27 & 1.24 & 0.20 \\
\hline VC5 & 59.31 & 0.89 & 15.31 & 4.40 & 3.99 & 0.35 & 3.56 & 5.85 & 5.50 & 0.67 & 0.19 \\
\hline VC6 & 55.62 & 0.87 & 15.49 & 3.77 & 4.16 & 0.29 & 3.50 & 9.80 & 6.10 & 0.21 & 0.19 \\
\hline VC7 & 47.54 & 0.86 & 17.95 & 5.57 & 4.33 & 0.24 & 3.45 & 13.43 & 4.57 & 1.88 & 0.19 \\
\hline VC8 & 54.46 & 0.84 & 15.95 & 3.92 & 4.50 & 0.19 & 3.08 & 7.45 & 7.29 & 2.12 & 0.19 \\
\hline VC9 & 52.85 & 0.82 & 17.27 & 4.61 & 4.67 & 0.14 & 3.35 & 7.46 & 7.89 & 0.76 & 0.19 \\
\hline VC10 & 54.69 & 0.81 & 15.77 & 4.27 & 4.15 & 0.09 & 3.30 & 6.07 & 6.28 & 4.39 & 0.19 \\
\hline VC11 & 52.15 & 0.79 & 17.48 & 4.24 & 5.01 & 0.03 & 3.24 & 9.87 & 4.04 & 2.96 & 0.19 \\
\hline VC12 & 54.00 & 0.78 & 16.91 & 5.36 & 5.18 & 0.02 & 3.19 & 7.28 & 2.10 & 5.00 & 0.19 \\
\hline VC13 & 54.46 & 1.63 & 16.78 & 3.92 & 2.33 & 0.79 & 4.00 & 11.34 & 3.12 & 1.42 & 0.20 \\
\hline VC14 & 50.31 & 0.81 & 17.15 & 1.65 & 5.05 & 0.02 & 3.23 & 10.56 & 8.95 & 2.07 & 0.19 \\
\hline VC15 & 56.54 & 0.80 & 16.15 & 3.94 & 5.24 & 0.32 & 3.18 & 6.48 & 5.04 & 2.12 & 0.19 \\
\hline VC16 & 56.08 & 0.79 & 15.35 & 2.14 & 5.43 & 0.20 & 3.13 & 6.67 & 9.24 & 0.79 & 0.19 \\
\hline VC17 & 54.00 & 0.78 & 16.92 & 3.99 & 5.62 & 0.19 & 3.07 & 7.78 & 3.28 & 4.19 & 0.19 \\
\hline VC18 & 59.08 & 0.77 & 12.45 & 3.48 & 5.81 & 0.19 & 3.02 & 7.60 & 4.46 & 2.96 & 0.19 \\
\hline VC19 & 53.08 & 0.75 & 17.20 & 4.56 & 6.00 & 0.19 & 2.96 & 7.60 & 6.17 & 1.30 & 0.19 \\
\hline VC20 & 52.85 & 0.74 & 16.42 & 5.57 & 6.19 & 0.19 & 2.91 & 7.43 & 6.46 & 1.05 & 0.20 \\
\hline VC21 & 50.31 & 0.73 & 18.04 & 1.36 & 6.38 & 0.91 & 2.85 & 12.40 & 5.85 & 0.98 & 0.20 \\
\hline $\mathrm{VC} 22$ & 51.00 & 0.71 & 17.83 & 1.87 & 6.57 & 0.92 & 2.80 & 14.10 & 2.99 & 1.02 & 0.19 \\
\hline VC23 & 54.00 & 0.70 & 16.09 & 4.40 & 6.76 & 0.93 & 2.74 & 9.28 & 3.27 & 1.64 & 0.19 \\
\hline $\mathrm{VC} 24$ & 45.92 & 0.84 & 18.42 & 5.86 & 6.95 & 0.94 & 2.69 & 14.31 & 2.99 & 0.89 & 0.19 \\
\hline VC25 & 51.23 & 0.68 & 16.89 & 5.86 & 7.13 & 0.24 & 2.64 & 11.00 & 3.27 & 0.87 & 0.19 \\
\hline VC26 & 46.85 & 0.67 & 19.09 & 5.70 & 7.32 & 0.19 & 2.58 & 11.06 & 5.50 & 0.86 & 0.19 \\
\hline $\mathrm{VC} 27$ & 52.38 & 0.65 & 17.41 & 4.69 & 7.51 & 0.14 & 2.53 & 7.54 & 6.10 & 0.84 & 0.20 \\
\hline VC28 & 54.23 & 0.64 & 16.02 & 4.35 & 7.70 & 0.09 & 2.47 & 8.90 & 4.57 & 0.82 & 0.19 \\
\hline VC29 & 54.46 & 0.63 & 16.78 & 5.28 & 7.89 & 0.03 & 2.42 & 4.26 & 7.29 & 0.76 & 0.19 \\
\hline VC30 & 59.31 & 0.62 & 15.31 & 3.43 & 8.08 & 0.15 & 2.36 & 2.58 & 3.58 & 4.39 & 0.18 \\
\hline
\end{tabular}


151 The studied samples exhibit variability in crystallinity and in mineralogical composition but, in 152 general, can be characterized as subvolcanic andesitic porphyrites. Plagioclase and clinopyroxene 153 are the main minerals in all samples analysed, showing variations in their mineral contents (Table 154 2) and grain sizes (Table 3). The equivalent quartz content (EQC) was determined according to 155 Thuro (1997). A suggested equation is shown in Eq. 1:

$156 E Q C=\sum A_{i} \cdot R_{i}$

157 where $A_{i}$ is the mineral amount (\%) and $R_{i}$ is the Rosiwal abrasiveness value for each mineral, 158 respectively.

159 In hand specimens, the Bombolí tunnel andesites exhibit a seriate porphyritic texture, with visible 160 phenocryst of plagioclases surrounded by a greenish grey fine-grained matrix. Polished thin 161 sections show largely euhedral to subhedral plagioclase phenocrysts, scanty subhedral 162 clinopyroxenes (between 5-13\% of phenocrists) and occasional hornblende phenocrists, with 163 opaques as accessory minerals (Fig. 4). Plagioclase crystals are generally unaltered and exhibit the 164 characteristic lamellar twinning, even some phenocrysts are partially resorbed. Some of the 165 plagioclase crystals show chemical zoning. Clinopyroxene phenocrystals are partially replaced by 166 chlorite. Small microlites of plagioclase and clinopyroxenes are embedded in a dark, glassy 167 groundmass.

\subsection{Cerchar abrasivity index, physical and mechanical properties}

169 Cerchar abrasivity index results obtained for each tested specimen are listed on Table 3. Unit

170 weight of the studied intact rock was found to be $25.4 \mathrm{kN} / \mathrm{m}^{3}$ for the TB samples, $24.9 \mathrm{kN} / \mathrm{m}^{3}$ for 171 the TT samples and $24.7 \mathrm{kN} / \mathrm{m}^{3}$ for the VC samples. Regarding uniaxial compression strength, tests 172 gave an average value of $35 \mathrm{MPa}$ for the TB samples, $25 \mathrm{MPa}$ for the TT samples and $30 \mathrm{MPa}$ for 173 the VC samples. Tensile strength was set to $10 \mathrm{MPa}$ in the case of TB samples, $9 \mathrm{MPa}$ in the case of 174 TT samples and $8 \mathrm{MPa}$ in the case of VC samples. 
Table 2. Modal compounds of the studied andesitic samples.

\begin{tabular}{|c|c|c|c|c|c|c|}
\hline \multirow{2}{*}{ Sample } & \multicolumn{5}{|c|}{ Modal compounds (\%) } & \multirow{2}{*}{ EQC $(\%)$} \\
\hline & Plagioclase & Clinopyroxene & Amphibole & Iron Ore & Cryptocrystalline material & \\
\hline TB1 & 59.7 & 17.9 & 3.0 & 0.0 & 19.4 & 28.3 \\
\hline TB2 & 71.9 & 16.1 & 2.2 & 0.1 & 9.7 & 30.3 \\
\hline TB3 & 71.4 & 16.0 & 1.6 & 1.0 & 10.0 & 30.2 \\
\hline TB4 & 62.0 & 23.1 & 5.5 & 0.0 & 9.4 & 29.6 \\
\hline TB5 & 67.4 & 18.3 & 5.4 & 0.1 & 8.8 & 29.8 \\
\hline TB6 & 53.8 & 33.2 & 3.0 & 0.0 & 10.0 & 29.6 \\
\hline TB7 & 57.1 & 29.2 & 3.7 & 0.0 & 10.0 & 29.6 \\
\hline TB8 & 76.8 & 7.1 & 1.9 & 2.2 & 12.0 & 29.8 \\
\hline TB9 & 71.5 & 12.6 & 2.6 & 1.4 & 11.9 & 29.7 \\
\hline TB10 & 68.8 & 15.8 & 3.3 & 0.0 & 12.1 & 29.7 \\
\hline TB11 & 65.3 & 18.9 & 3.6 & 0.2 & 12.0 & 29.5 \\
\hline TB 12 & 64.9 & 17.6 & 3.4 & 2.1 & 12.0 & 29.2 \\
\hline TB13 & 60.7 & 21.6 & 5.9 & 1.0 & 10.8 & 29.1 \\
\hline TB14 & 57.5 & 23.7 & 5.6 & 2.4 & 10.8 & 28.8 \\
\hline TB 15 & 58.6 & 24.6 & 5.5 & 0.1 & 11.2 & 29.2 \\
\hline TB 16 & 63.7 & 21.0 & 3.9 & 1.4 & 10.0 & 29.5 \\
\hline TB17 & 59.9 & 26.0 & 4.1 & 0.2 & 9.8 & 29.6 \\
\hline TB18 & 55.8 & 28.3 & 4.2 & 0.3 & 11.3 & 29.2 \\
\hline TB19 & 51.6 & 32.6 & 3.7 & 2.0 & 10.1 & 29.1 \\
\hline TB20 & 58.6 & 26.9 & 3.4 & 1.1 & 10.0 & 29.5 \\
\hline TB21 & 57.1 & 26.6 & 5.5 & 0.0 & 10.8 & 29.2 \\
\hline TB22 & 62.5 & 21.1 & 4.6 & 0.0 & 11.8 & 29.3 \\
\hline TB23 & 56.6 & 28.5 & 3.0 & 0.0 & 11.9 & 29.4 \\
\hline TB24 & 50.7 & 34.6 & 3.7 & 0.0 & 11.0 & 29.3 \\
\hline TB25 & 74.6 & 9.4 & 1.9 & 2.1 & 12.0 & 29.7 \\
\hline TB26 & 66.2 & 19.9 & 2.6 & 0.9 & 10.4 & 29.8 \\
\hline TB27 & 55.5 & 31.8 & 3.0 & 0.0 & 9.7 & 29.7 \\
\hline TT1 & 57.9 & 29.1 & 3.0 & 0.0 & 10.0 & 29.7 \\
\hline TT2 & 52.0 & 33.6 & 4.1 & 0.0 & 10.3 & 29.3 \\
\hline TT3 & 57.2 & 28.5 & 4.3 & 0.0 & 10.0 & 29.5 \\
\hline TT4 & 58.2 & 26.5 & 3.7 & 0.0 & 11.6 & 29.4 \\
\hline TT5 & 58.3 & 28.3 & 3.3 & 0.0 & 10.1 & 29.7 \\
\hline TT6 & 52.7 & 34.3 & 3.0 & 0.0 & 10.0 & 29.6 \\
\hline TT7 & 67.2 & 18.3 & 2.7 & 1.0 & 10.8 & 29.8 \\
\hline TT8 & 48.8 & 37.6 & 2.8 & 0.1 & 10.7 & 29.3 \\
\hline TT9 & 58.3 & 29.2 & 2.8 & 0.1 & 9.6 & 29.8 \\
\hline TT10 & 51.0 & 37.1 & 0.6 & 0.3 & 11.0 & 29.6 \\
\hline TT11 & 54.1 & 30.5 & 3.9 & 0.1 & 11.4 & 29.2 \\
\hline TT12 & 55.9 & 29.9 & 4.0 & 0.3 & 9.9 & 29.5 \\
\hline TT13 & 60.2 & 24.5 & 3.7 & 0.0 & 11.6 & 29.4 \\
\hline TT14 & 61.2 & 25.3 & 3.4 & 0.0 & 10.1 & 29.7 \\
\hline TT15 & 53.2 & 31.4 & 5.5 & 0.0 & 9.9 & 29.2 \\
\hline TT16 & 50.5 & 34.1 & 4.6 & 0.0 & 10.8 & 29.1 \\
\hline VC1 & 56.8 & 30.9 & 1.5 & 0.0 & 10.8 & 29.8 \\
\hline VC2 & 49.4 & 36.0 & 3.3 & 0.3 & 11.0 & 29.2 \\
\hline VC3 & 63.8 & 22.7 & 1.9 & 0.0 & 11.6 & 29.8 \\
\hline VC4 & 51.8 & 34.0 & 4.0 & 0.1 & 10.1 & 29.4 \\
\hline VC5 & 57.4 & 27.8 & 3.7 & 2.2 & 8.9 & 29.4 \\
\hline VC6 & 53.8 & 30.6 & 2.4 & 1.4 & 11.8 & 29.2 \\
\hline VC7 & 46.0 & 39.3 & 5.3 & 0.0 & 9.4 & 29.1 \\
\hline VC8 & 56.7 & 27.8 & 4.4 & 0.0 & 11.1 & 29.3 \\
\hline VC9 & 59.4 & 25.6 & 3.0 & 0.0 & 12.0 & 29.4 \\
\hline VC10 & 52.9 & 33.5 & 3.6 & 0.2 & 9.8 & 29.5 \\
\hline VC11 & 50.5 & 37.5 & 1.9 & 0.0 & 10.1 & 29.6 \\
\hline VC12 & 56.0 & 35.7 & 1.6 & 0.0 & 6.7 & 30.4 \\
\hline VC13 & 55.7 & 34.4 & 2.7 & 0.0 & 7.2 & 30.1 \\
\hline VC14 & 52.4 & 34.5 & 2.8 & 0.0 & 10.3 & 29.5 \\
\hline VC15 & 54.7 & 31.4 & 3.8 & 0.0 & 10.1 & 29.5 \\
\hline VC16 & 54.3 & 34.8 & 0.6 & 0.0 & 10.3 & 29.9 \\
\hline VC17 & 53.3 & 35.8 & 1.2 & 0.7 & 9.0 & 29.9 \\
\hline VC18 & 57.2 & 28.6 & 4.0 & 1.6 & 8.6 & 29.5 \\
\hline VC19 & 51.4 & 35.6 & 2.9 & 0.0 & 10.1 & 29.5 \\
\hline VC20 & 51.2 & 36.1 & 2.2 & 0.6 & 9.9 & 29.5 \\
\hline $\mathrm{VC} 21$ & 51.7 & 31.6 & 1.6 & 1.9 & 13.2 & 28.9 \\
\hline $\mathrm{VC} 22$ & 49.4 & 36.1 & 2.2 & 1.5 & 10.8 & 29.2 \\
\hline $\mathrm{VC} 23$ & 66.7 & 25.6 & 3.0 & 0.0 & 4.7 & 30.8 \\
\hline $\mathrm{VC} 24$ & 44.5 & 40.9 & 3.3 & 0.2 & 11.1 & 29.1 \\
\hline $\mathrm{VC} 25$ & 49.6 & 36.9 & 1.9 & 2.2 & 9.4 & 29.4 \\
\hline VC26 & 45.3 & 42.8 & 1.6 & 1.4 & 8.9 & 29.5 \\
\hline VC27 & 54.2 & 30.9 & 2.7 & 0.4 & 11.8 & 29.3 \\
\hline VC28 & 52.5 & 35.0 & 2.8 & 0.0 & 9.7 & 29.6 \\
\hline VC29 & 60.3 & 25.1 & 3.7 & 0.9 & 10.0 & 29.5 \\
\hline VC30 & 57.4 & 30.1 & 3.2 & 0.0 & 9.3 & 29.8 \\
\hline
\end{tabular}


Table 3. Grain size and Cerchar abrasivity index (CAI).

\begin{tabular}{|c|c|c|c|c|}
\hline \multirow{2}{*}{ Sample } & \multicolumn{3}{|c|}{ Grain Size $(\mathrm{mm})$} & \multirow{2}{*}{ CAI } \\
\hline & Plagioclase & Clinopyroxene & Amphibole & \\
\hline TB1 & 1.04 & 0.64 & 0.87 & 2.30 \\
\hline TB2 & 1.20 & 0.80 & 0.61 & 2.82 \\
\hline TB3 & 1.33 & 0.33 & 0.66 & 3.02 \\
\hline TB4 & 1.07 & 0.58 & 0.87 & 2.43 \\
\hline TB5 & 1.08 & 0.48 & 0.45 & 2.69 \\
\hline TB6 & 0.80 & 0.45 & 0.53 & 2.11 \\
\hline TB7 & 0.90 & 1.00 & 0.62 & 2.20 \\
\hline TB8 & 1.37 & 0.66 & 0.33 & 3.10 \\
\hline TB9 & 1.30 & 0.46 & 0.27 & 2.86 \\
\hline TB10 & 1.20 & 0.67 & 0.62 & 2.65 \\
\hline TB11 & 1.11 & 0.63 & 0.87 & 2.56 \\
\hline TB12 & 1.13 & 0.31 & 0.45 & 2.50 \\
\hline TB13 & 1.03 & 1.10 & 0.53 & 2.38 \\
\hline TB14 & 0.99 & 0.34 & 0.33 & 2.39 \\
\hline TB15 & 0.96 & 0.70 & 0.27 & 2.30 \\
\hline TB16 & 1.24 & 0.90 & 0.61 & 2.50 \\
\hline TB17 & 1.04 & 0.30 & 0.66 & 2.31 \\
\hline TB18 & 0.89 & 0.50 & 0.71 & 2.19 \\
\hline TB19 & 0.85 & 0.32 & 0.43 & 1.99 \\
\hline TB20 & 1.00 & 0.18 & 0.36 & 2.30 \\
\hline TB21 & 1.06 & 0.68 & 0.29 & 2.24 \\
\hline TB22 & 1.15 & 0.43 & 0.27 & 2.45 \\
\hline TB23 & 0.93 & 0.19 & 0.62 & 2.18 \\
\hline TB24 & 0.83 & 0.20 & 0.83 & 1.99 \\
\hline TB25 & 1.33 & 0.46 & 0.29 & 3.25 \\
\hline TB26 & 1.21 & 0.78 & 0.72 & 2.67 \\
\hline TB27 & 0.92 & 0.50 & 0.75 & 2.31 \\
\hline TT1 & 1.00 & 0.36 & 0.76 & 2.23 \\
\hline TT2 & 1.26 & 0.57 & 0.18 & 2.33 \\
\hline TT3 & 1.11 & 0.43 & 0.20 & 2.56 \\
\hline TT4 & 1.22 & 0.56 & 0.45 & 2.42 \\
\hline TT5 & 1.22 & 0.70 & 0.33 & 2.61 \\
\hline TT6 & 1.04 & 0.30 & 0.22 & 2.36 \\
\hline TT7 & 1.33 & 0.48 & 0.27 & 3.01 \\
\hline TT8 & 0.93 & 0.17 & 0.23 & 1.88 \\
\hline TT9 & 1.18 & 0.20 & 0.56 & 2.61 \\
\hline TT10 & 1.01 & 0.34 & 0.76 & 2.30 \\
\hline TT11 & 1.18 & 0.60 & 0.39 & 2.42 \\
\hline TT12 & 1.09 & 0.55 & 0.42 & 2.51 \\
\hline TT13 & 1.03 & 0.55 & 0.87 & 2.32 \\
\hline TT14 & 1.05 & 0.57 & 0.76 & 2.36 \\
\hline TT15 & 1.07 & 0.59 & 0.37 & 2.38 \\
\hline TT16 & 0.89 & 0.44 & 0.75 & 2.26 \\
\hline VC1 & 0.98 & 0.21 & 0.38 & 2.19 \\
\hline VC2 & 1.04 & 0.12 & 0.57 & 2.21 \\
\hline VC3 & 1.06 & 0.15 & 0.76 & 2.46 \\
\hline VC4 & 1.05 & 0.18 & 0.36 & 2.32 \\
\hline VC5 & 1.17 & 0.34 & 0.65 & 2.57 \\
\hline VC6 & 1.03 & 0.71 & 0.44 & 2.41 \\
\hline VC7 & 0.96 & 0.68 & 0.36 & 2.06 \\
\hline VC8 & 1.01 & 0.41 & 0.32 & 2.36 \\
\hline VC9 & 1.08 & 0.69 & 0.29 & 2.29 \\
\hline VC10 & 1.07 & 0.76 & 0.17 & 2.37 \\
\hline VC11 & 1.02 & 0.63 & 0.62 & 2.26 \\
\hline VC12 & 1.06 & 0.65 & 0.67 & 2.34 \\
\hline VC13 & 1.07 & 0.75 & 0.61 & 2.36 \\
\hline VC14 & 0.98 & 0.64 & 0.46 & 2.18 \\
\hline VC15 & 1.11 & 0.56 & 0.36 & 2.45 \\
\hline VC16 & 1.10 & 1.20 & 0.29 & 2.43 \\
\hline VC17 & 1.06 & 0.21 & 0.27 & 2.34 \\
\hline VC18 & 1.16 & 0.34 & 0.62 & 2.56 \\
\hline VC19 & 1.04 & 0.54 & 0.77 & 2.30 \\
\hline VC20 & 1.03 & 0.43 & 0.56 & 2.29 \\
\hline $\mathrm{VC} 21$ & 0.98 & 0.53 & 0.59 & 2.18 \\
\hline $\mathrm{VC} 22$ & 1.00 & 0.67 & 0.61 & 2.21 \\
\hline VC23 & 1.06 & 0.78 & 0.58 & 2.34 \\
\hline $\mathrm{VC} 24$ & 0.91 & 0.45 & 0.19 & 1.99 \\
\hline $\mathrm{VC} 25$ & 1.02 & 0.56 & 0.27 & 2.22 \\
\hline VC26 & 0.93 & 0.34 & 0.45 & 2.03 \\
\hline $\mathrm{VC} 27$ & 1.02 & 0.58 & 0.23 & 2.27 \\
\hline $\mathrm{VC} 28$ & 1.06 & 0.63 & 0.15 & 2.35 \\
\hline VC29 & 1.09 & 0.76 & 0.38 & 2.36 \\
\hline VC30 & 1.15 & 0.64 & 0.23 & 2.57 \\
\hline
\end{tabular}




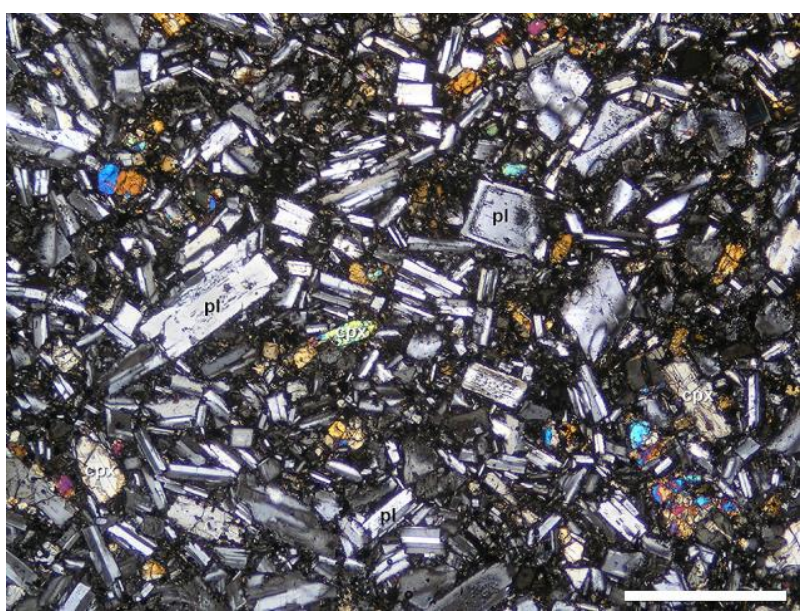

Fig. 4. Representative photomicrograph in cross-polarized light of a porphyritic andesite sample from the project area (Bombolí hacienda, Ecuador) showing elongate, euhedral phenocrysts of plagioclase (grey interference colours, multiple twinning), clinopyroxene crystals (higher-order interference colours) and plagioclase microlites set in a glassy groundmass (black areas, optically isotropic). Clinopyroxene phenocrysts are partially altered and replaced by chlorite. Abbreviations: pl, plagioclase; cpx, clinopyroxene. Scale bar $=1 \mathrm{~mm}$.

\subsection{Statistical summary of the results}

Table 4 shows a statistical summary of the results obtained. Average, standard deviation, coefficient of variation and minimum and maximum values are listed for the chemical compounds and the petrographical properties of the andesitic samples studied, as well as for the Cerchar Abrasivity Index $(C A I)$. As may be observed, $C A I$ shows little variation, with a coefficient of variation of about a 10\%, while in general chemical compounds and petrographical properties exhibit more variability (with a coefficient of variation larger than a 50\% in some cases).

Regarding the relation between the $C A I$ and the grain size, if the test is scratch $10 \mathrm{~mm}$, it turns out that on average: (i) $57.4 \%$ will be plagioclase crystals with an average size of $1.07 \mathrm{~mm}$; (ii) $28.3 \%$ will be pyroxene crystals with an average size of $0.52 \mathrm{~mm}$; (iii) $3.3 \%$ amphibole crystals with average size of $0.49 \mathrm{~mm}$; (iv) $0.5 \%$ iron that we do not know its size or is rather dispersed; (v) $10.5 \%$ matrix. Hence, there is about $30 \%$ chance of scratching amphiboles and clinopyroxenes, approximately $60 \%$ of plagioclase and $10 \%$ of matrix, which is obvious from the distribution of minerals in the thin sections. 
Table 4.Statistical summary.

\begin{tabular}{|c|c|c|c|c|c|}
\hline & Average & $\begin{array}{l}\text { Standard } \\
\text { deviation }\end{array}$ & $\begin{array}{l}\text { Coefficient of } \\
\text { variation }(\%)\end{array}$ & $\begin{array}{l}\text { Minimum } \\
\text { value }\end{array}$ & $\begin{array}{l}\text { Maximum } \\
\text { value }\end{array}$ \\
\hline \multicolumn{6}{|l|}{ Chemical compounds (\%) } \\
\hline $\mathrm{SiO}_{2}$ & 55.39 & 5.29 & 9.55 & 43.38 & 75.00 \\
\hline $\mathrm{TiO}_{2}$ & 0.81 & 0.24 & 29.63 & 0.39 & 1.63 \\
\hline $\mathrm{Al}_{2} \mathrm{O}_{3}$ & 15.68 & 2.75 & 17.54 & 2.98 & 11.14 \\
\hline $\mathrm{Fe}_{2} \mathrm{O}_{3}$ & 4.12 & 1.32 & 32.04 & 1.36 & 7.29 \\
\hline $\mathrm{FeO}$ & 4.98 & 2.76 & 55.42 & 0.35 & 11.69 \\
\hline $\mathrm{MnO}$ & 0.65 & 0.62 & 95.38 & 0.02 & 2.35 \\
\hline $\mathrm{MgO}$ & 3.03 & 0.91 & 30.03 & 1.33 & 4.72 \\
\hline $\mathrm{CaO}$ & 9.39 & 3.36 & 35.78 & 0.11 & 18.24 \\
\hline $\mathrm{Na}_{2} \mathrm{O}$ & 3.49 & 1.86 & 53.30 & 0.17 & 9.24 \\
\hline $\mathrm{K}_{2} \mathrm{O}$ & 2.26 & 1.31 & 57.96 & 0.21 & 5.00 \\
\hline $\mathrm{P}_{2} \mathrm{O}_{5}$ & 0.19 & 0.03 & 15.79 & 0.08 & 0.32 \\
\hline \multicolumn{6}{|l|}{ Modal compounds (\%) } \\
\hline Plagioclase & 57.44 & 6.80 & 11.84 & 44.50 & 76.80 \\
\hline Clinopyroxene & 28.33 & 7.48 & 26.40 & 7.10 & 42.80 \\
\hline Amphibole & 3.26 & 1.19 & 36.50 & 0.60 & 5.90 \\
\hline Iron Ore & 0.49 & 0.73 & 148.97 & 0.00 & 2.40 \\
\hline Cryptocrystalline material & 10.47 & 1.67 & 15.95 & 4.70 & 19.40 \\
\hline$E Q C(\%)$ & 29.51 & 0.36 & 1.22 & 28.30 & 30.80 \\
\hline \multicolumn{6}{|l|}{ Grain size } \\
\hline Plagioclase & 1.07 & 0.12 & 11.21 & 0.80 & 1.37 \\
\hline Clinopyroxene & 0.52 & 0.22 & 42.31 & 0.12 & 1.20 \\
\hline Amphibole & 0.49 & 0.21 & 42.86 & 0.15 & 0.87 \\
\hline$C A I$ & 2.39 & 0.25 & 10.46 & 1.88 & 3.25 \\
\hline
\end{tabular}

200

Additionally, Table 5 displays the correlation matrix between CAI, minerals and EQC. From the observation of this matrix, it follows that the $C A I$ values with the plagioclase are logical in content and size of the crystal. Also with amphibole, but it does not have seemingly sense the variation that the clinopiroxeno presents. However, although the value of the correlation coefficient $(0.78)$ is low, the most significant is the negative sign. This indicates that the greater existence of clinopyroxene lower $C A I$.

\section{Analysis and Discussion}

Regression analyses were conducted to study the influence of the different chemical compounds and petrographical properties of the andesitic samples on CAI. The statistical software STATGRAPHICS Centurion XVI v16.2.04 (StatPoint Technologies, 2009) was used to perform the statistical analyses. 
Table 5. Correlation matrix between CAI. minerals and EQC.

\begin{tabular}{lllllll}
\hline & $\begin{array}{l}\text { Cerchar } \\
(\mathrm{CAI})\end{array}$ & $\begin{array}{l}\text { Plagioclase } \\
(\%)\end{array}$ & $\begin{array}{l}\text { Clinopiroxene } \\
(\%)\end{array}$ & $\begin{array}{l}\text { Amphibole } \\
(\%)\end{array}$ & $\begin{array}{l}\text { Iron } \\
\text { ore }(\%)\end{array}$ & $\begin{array}{l}\text { Cryptocrystalline } \\
\text { material }(\%)\end{array}$ \\
\hline $\begin{array}{l}\text { Plagioclase }(\mathrm{mm}) \\
\text { Plagioclase }(\%)\end{array}$ & 0.87 & 0.83 & 1 & & & \\
$\begin{array}{l}\text { Clinopiroxene } \\
(\mathrm{mm})\end{array}$ & 0.14 & & & & \\
$\begin{array}{l}\text { Clinopiroxene (\%) } \\
\text { Amphibole (mm) }\end{array}$ & -0.78 & -0.96 & 1 & & & \\
$\begin{array}{l}\text { Amphibole }(\%) \\
\text { Iron ore }(\%)\end{array}$ & -0.11 & 0 & -0.15 & 1 & & \\
$\begin{array}{l}\text { Cryptocrystalline } \\
\text { material }(\%)\end{array}$ & 0.29 & 0.2 & -0.27 & 0.15 & 1 & \\
EQC $(\%)$ & 0.04 & 0.11 & -0.33 & -0.01 & 0.07 & 1 \\
\hline
\end{tabular}

A simple linear regression was carried out between $C A I$ and the every chemical compound / petrographical property. Those regressions may be mathematically transcribed as:

$$
C A I=\alpha \cdot X_{i}+\beta
$$

where $X_{i}$ indicates the chemical compound / petrographical property (e.g. $\mathrm{SiO}_{2}, \mathrm{FeO}$, Plagioclase content, Amphibole grain size) and $\alpha$ and $\beta$ are the linear regression coefficients (being the former the slope and the latter the intercept) which are listed in Table 6. Besides, that table contains, for each analysis, the coefficient of determination $\left(R^{2}\right)$ as well as the residuals $p$-value (probability value). Assuming 5\% as significance level (as is commonly accepted), results with a p-value lower than 0.05 might be considered to be statistically significant at a confidence level of $95 \%$.

The higher correlation between $C A I$ and chemical compounds were found for $\mathrm{SiO}_{2}$ (coefficient of determination of $88.3 \%)$ and $\mathrm{Al}_{2} \mathrm{O}_{3}(66.8 \%)$. Fig. 5 displays graphically these relations. It should be noted that according to $p$-values chemical compounds $\mathrm{TiO}_{2}, \mathrm{MnO}, \mathrm{MgO}, \mathrm{Na}_{2} \mathrm{O}$ and $\mathrm{P}_{2} \mathrm{O}_{5}$ appear not to be statistically significant (even though, in the case of $\mathrm{P}_{2} \mathrm{O}_{5}$ coefficient of determination is considerable higher compared to the other mentioned compounds). Results obtained could be compared with what was recently reported by Er and Tugrul (2016b), who studied the influence of chemical compounds on $C A I$ for the granitic rocks of Turkey. Those authors found that $\mathrm{SiO}_{2}, \mathrm{Al}_{2} \mathrm{O}_{3}$ and $\mathrm{Fe}_{2} \mathrm{O}_{3}$ were the compounds which presented the highest correlation with CAI, reaching a $R^{2}$ 
231 value of around a $42 \%$, a similar value to that obtained here for $\mathrm{Fe}_{2} \mathrm{O}_{3}$, but rather lower for $\mathrm{SiO}_{2}$ and $232 \mathrm{Al}_{2} \mathrm{O}_{3}$ when compared with the results presented on this paper. This difference may be put down to 233 the actual modal difference existing between granitic and andesitic rocks.

234 Table 6. Simple linear regression results.

\begin{tabular}{lllll}
\hline & $\alpha$ & $\beta$ & $\mathrm{R}^{2}$ & $\mathrm{p}$-value \\
\hline Chemical compounds & & & & \\
$\mathrm{SiO}_{2}$ & 0.0448 & -0.0886 & 0.883 & 0.000 \\
$\mathrm{TiO}_{2}$ & 0.1159 & 2.2972 & 0.012 & 0.349 \\
$\mathrm{Al}_{2} \mathrm{O}_{3}$ & -0.0749 & 3.5651 & 0.668 & 0.000 \\
$\mathrm{Fe}_{2} \mathrm{O}_{3}$ & -0.1328 & 2.9382 & 0.481 & 0.000 \\
$\mathrm{FeO}$ & -0.0261 & 2.5209 & 0.082 & 0.014 \\
$\mathrm{MnO}$ & -0.0822 & 2.4444 & 0.041 & 0.087 \\
$\mathrm{MgO}$ & 0.0316 & 2.2947 & 0.013 & 0.337 \\
$\mathrm{CaO}$ & -0.0225 & 2.6019 & 0.090 & 0.010 \\
$\mathrm{Na} 2 \mathrm{O}$ & -0.0271 & 2.4853 & 0.039 & 0.090 \\
$\mathrm{~K} 2 \mathrm{O}$ & 0.0516 & 2.2742 & 0.072 & 0.022 \\
$\mathrm{P}_{2} \mathrm{O}_{5}$ & 0.5879 & 2.2802 & 0.499 & 0.552 \\
Modal compounds & & & & \\
$\mathrm{Plagioclase}$ & 0.0305 & 0.6360 & 0.688 & 0.000 \\
$\mathrm{Clinopyroxene}$ & -0.0261 & 3.1319 & 0.604 & 0.000 \\
$\mathrm{Amphibole}$ & -0.0242 & 2.4696 & 0.013 & 0.332 \\
Iron Ore & 0.0981 & 2.3422 & 0.082 & 0.014 \\
Cryptocrystalline material & 0.0062 & 2.3258 & 0.002 & 0.728 \\
EQC (\%) & 0.2839 & -5.9884 & 0.169 & 0.000 \\
Grain size & & & & \\
Plagioclase & 1.8243 & 0.4447 & 0.875 & 0.000 \\
Clinopyroxene & 0.1607 & 2.3067 & 0.020 & 0.232 \\
Amphibole & -0.0863 & 2.4327 & 0.005 & 0.553 \\
\hline
\end{tabular}
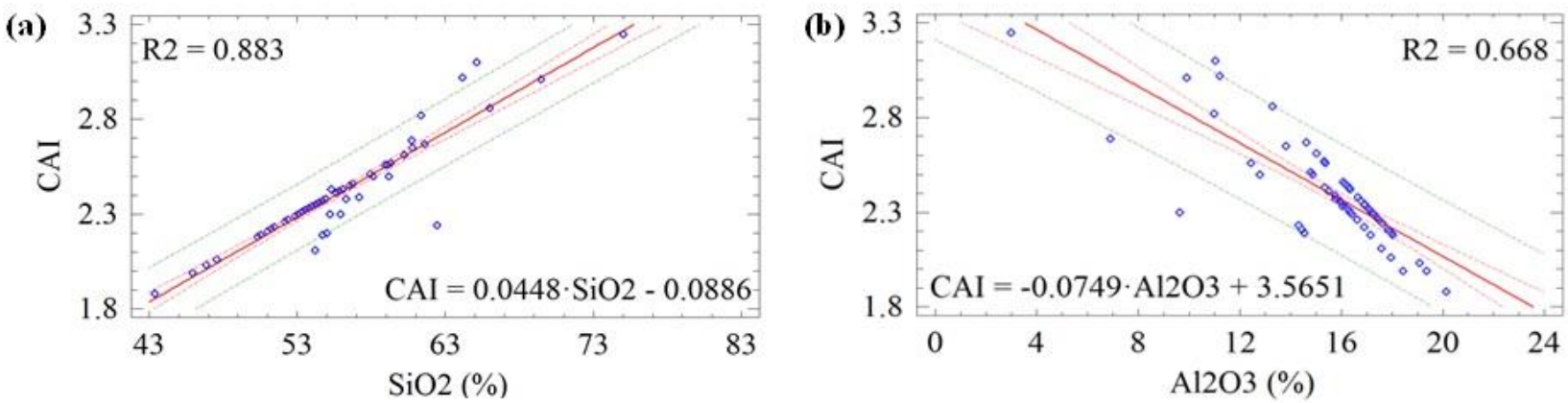

Fig. 5. Simple linear correlation on chemical compounds: a) relation between CAI and SiO2; b) relation between CAI 
Regarding petrographical properties (modal compounds and grain size), the higher correlation were 241 found for grain size - plagioclase (coefficient of determination of 87.5\%) and modal compound 242 plagioclase (68.8\%). Fig. 6 displays graphically these relations. According to p-values, minerals amphibole and cryptocrystalline material appear not to be statistically significant in terms of modal compound and minerals clinopyroxene and amphibole appear not to be statistically significant in terms of grain size. If results are compared with those obtained by Er and Tugrul (2016b), who also studied the influence of petrographical properties on CAI, no match is observed in this case. Granitic rocks studied by those authors had Quartz as the main modal compound, and correlation between $C A I$ and EQC produced a $R^{2}$ value of around $64 \%$, which is rather higher that what was obtained for the andesitic rocks studied in this paper $(16.9 \%)$. In this case, it is clear that the difference in the petrographical nature between granitic and andesitic rocks is the reason of such discrepancy in results. On the other hand, results obtained are in accordance with Alber (2008) who also established that there was no significant correlation between $C A I$ and $E Q C$.
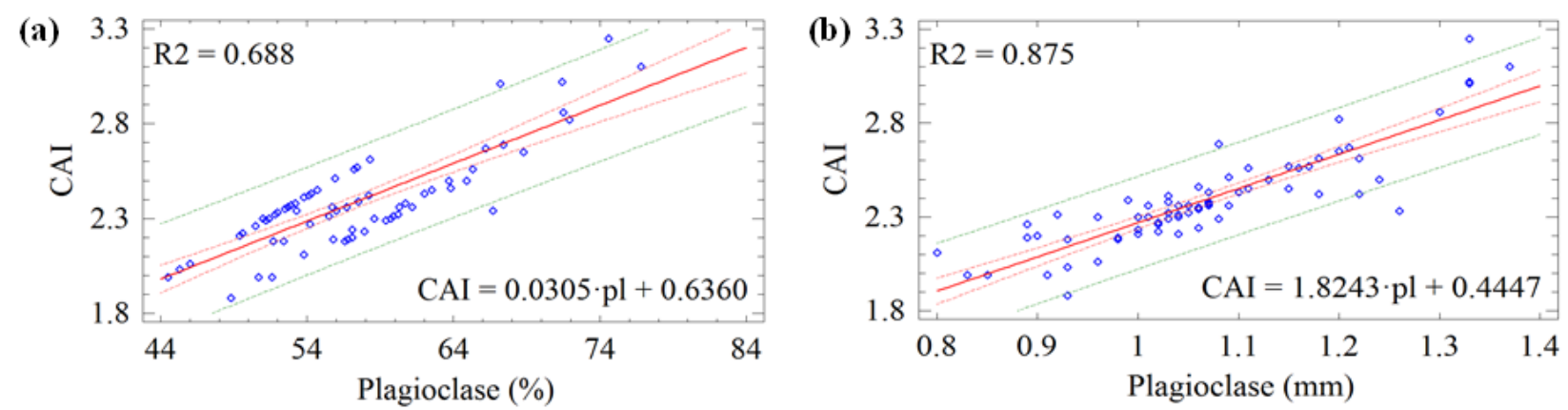

Fig. 6. Simple linear correlation on petrographical properties: a) relation between CAI and modal compound plagioclase; b) relation between CAI and grain size - plagioclase. Red and green dotted line indicate confidence interval and prediction interval, respectively, for a $95 \%$ level of significance.

With the aim of improving correlation, a linear multiregression analysis was conducted. Following that, $C A I$ may be expressed as: 
where $X_{i}$ indicates the chemical compound / petrographical property (e.g. $\mathrm{SiO}_{2}, \mathrm{FeO}$, Plagioclase 261 content, Amphibole grain size) and $\alpha_{i}$ and $\beta^{*}$ are the linear regression coefficients (being the former 262 the slope for each compound/property and the latter the intercept) which are listed in Table 7. 263 Besides, that table contains, for each analysis, the coefficient of determination $\left(R^{2}\right)$ as well as the 264 compound/property p-value. Assuming 5\% as significance level (as is commonly accepted), results 265 with a $p$-value lower than 0.05 might be considered to be statistically significant at a confidence 266 level of $95 \%$. Those compounds/properties that produced a $p$-value higher than 0.05 were removed 267 from the analysis, since those compounds/properties may be considered not to be statistically 268 significant.

Table 7. Multiregressions results.

\begin{tabular}{|c|c|c|c|c|}
\hline & $\alpha_{\mathrm{i}}$ & $\beta^{*}$ & $\mathrm{R}^{2}$ & p-value \\
\hline \multicolumn{5}{|l|}{ Chemical compounds } \\
\hline $\mathrm{SiO}_{2}$ & 0.0552 & -1.3191 & 0.924 & 0.000 \\
\hline $\mathrm{TiO}_{2}$ & - & & & - \\
\hline $\mathrm{Al}_{2} \mathrm{O}_{3}$ & - & & & - \\
\hline $\mathrm{Fe}_{2} \mathrm{O}_{3}$ & - & & & - \\
\hline $\mathrm{FeO}$ & 0.0261 & & & 0.000 \\
\hline $\mathrm{MnO}$ & - & & & - \\
\hline $\mathrm{MgO}$ & 0.0545 & & & 0.000 \\
\hline $\mathrm{CaO}$ & 0.0244 & & & 0.000 \\
\hline $\mathrm{Na}_{2} \mathrm{O}$ & 0.0223 & & & 0.000 \\
\hline $\mathrm{K}_{2} \mathrm{O}$ & 0.0223 & & & 0.000 \\
\hline $\mathrm{P}_{2} \mathrm{O}_{5}$ & - & & & 0.004 \\
\hline \multicolumn{5}{|l|}{ Modal compounds } \\
\hline Plagioclase & 0.0305 & 0.6360 & 0.688 & 0.000 \\
\hline Clinopyroxene & - & & & - \\
\hline Amphibole & - & & & - \\
\hline Iron Ore & - & & & - \\
\hline Cryptocrystalline material & - & & & - \\
\hline \multicolumn{5}{|l|}{ Grain size } \\
\hline Plagioclase & 1.8243 & 0.4447 & 0.875 & 0.000 \\
\hline Clinopyroxene & - & & & - \\
\hline Amphibole & - & & & - \\
\hline
\end{tabular}

When comparing results obtained using multiregression analysis with simple regression, it may be observed that the use of multiregression slightly improves the estimation of CAI based on chemical compounds. Coefficient of determination increases from $88.3 \%$ (best result obtained for simple 274 regression for $\mathrm{SiO}_{2}$ ) to $92.4 \%$, and in the correlation equation take part $\mathrm{SiO}_{2}, \mathrm{FeO}, \mathrm{MgO}, \mathrm{CaO}$, $275 \quad \mathrm{Na}_{2} \mathrm{O}$ and $\mathrm{K}_{2} \mathrm{O}$ compounds: 
277 It is interesting to note that $\mathrm{Al}_{2} \mathrm{O}_{3}$ resulted not to be statistically significant in this analysis, even 278 though that compound reached the second highest $R^{2}$ when performing the simple regression 279 analyses.

280 For the case of petrographical properties, every property except plagioclase (as modal compound 281 and as grain size) showed not to be statistically significant. Therefore, no improvement was 282 achieved by using multiregression analysis (note that values for $\alpha_{i}, \beta^{*}$ and $R^{2}$ are exactly the same as 283 those obtained with a simple linear regression for plagioclase).

284 Eventually, if a graph comparing $C A I$ vs. the modal compounds of the plagioclase and vs. the rest of 285 the compounds (clinopyroxene, amphibole and cryptocrystalline material) is made (Fig. 7), the 286 regression analyses conducted are confirmed. The correlation of $C A I$ with plagioclase is positive so 287 the more of this compound, the higher the value of CAI. On the other hand, the content of the other 288 compounds tend to lower the $C A I$, as was noted in the statistical summary of the results.

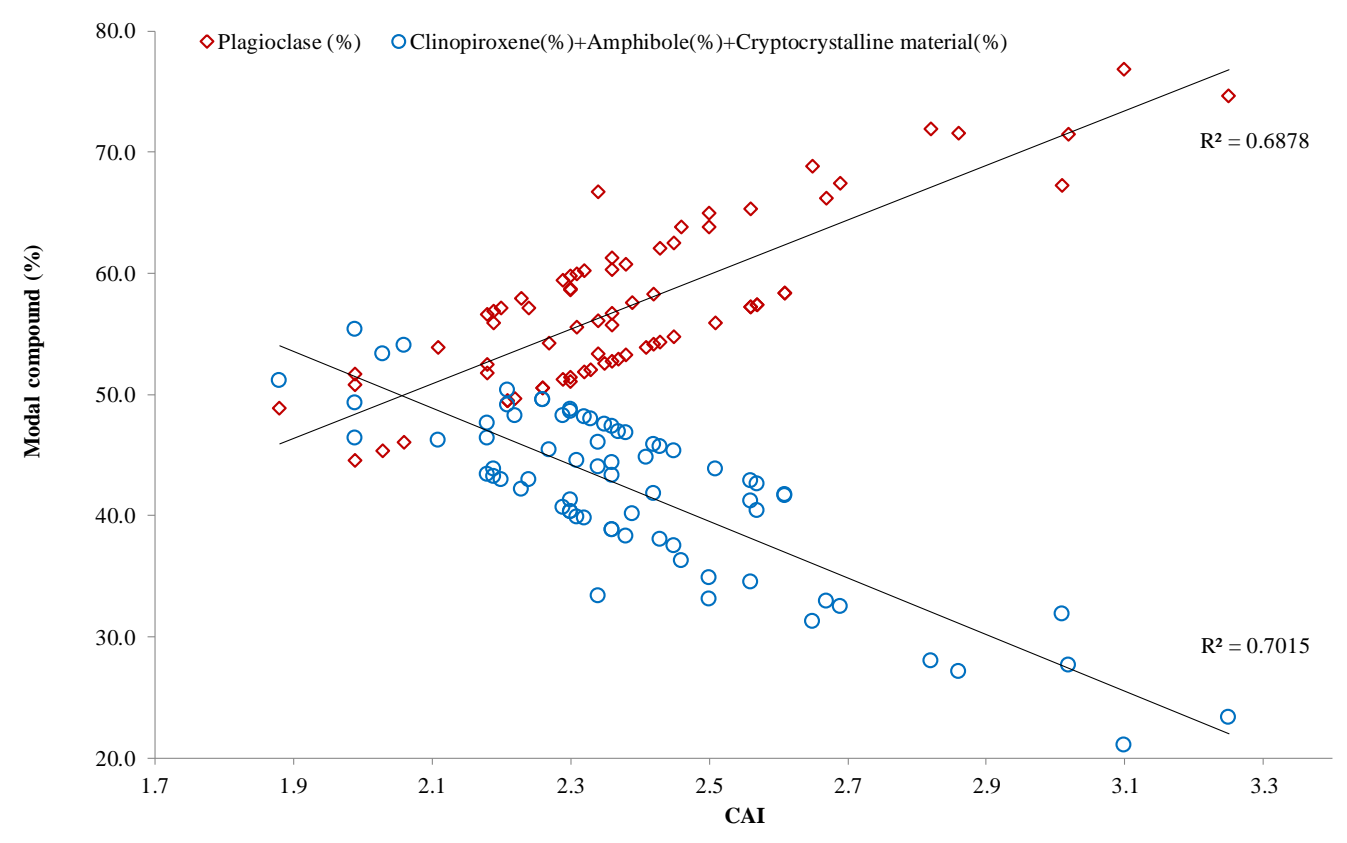




\section{Conclusion}

293 Relation between Cerchar Abrasivity Index (CAI) and the chemical compounds and petrographical properties (modal compounds and grain size) of a series of andesitic rocks samples coming from the central area of Ecuador was investigated. A total of 73 andesitic samples were subjected to XRF analyses to find their chemical compounds. Modal compounds and minerals grain size were obtained by preparing thin sections of each sample. CAI was computed by conducting a Cerchar abrasivity test on each sample. Density, uniaxial compression strength and tensile strength of the andesitic rock was also obtained to complete the geotechnical characterization of that material. Several regression analyses were performed with the aim of establishing the significance and relation that the different chemical compounds, the modal compounds and the minerals grain size might have on $C A I$.

303 From the results obtained it may be concluded:

a) The andesitic samples resulted to be composed of mainly plagioclase (nearly 60\%), with some content in clinopyroxenes (around 30\%) and some traces of amphibole and iron ore. Chemically, the samples mainly consist of $\mathrm{SiO}_{2}$ (ca. 50\%) with some content in $\mathrm{Al}_{2} \mathrm{O}_{3}$ and $\mathrm{CaO}$, and traces of other compounds such as $\mathrm{Fe}_{2} \mathrm{O}_{3}, \mathrm{MgO}$ and $\mathrm{P}_{2} \mathrm{O}_{5}$. Regarding physical and mechanical properties, density of andesitic rock samples was found to be about $25 \mathrm{kN} / \mathrm{m}^{3}$, uniaxial compression strength $177 \mathrm{MPa}$ and tensile strength $9 \mathrm{MPa}$. CAI achieved an average value of 2.39 .

b) The Cerchar tests showed that there is about $30 \%$ chance of scratching amphiboles and clinopyroxenes, approximately $60 \%$ of plagioclase and $10 \%$ of matrix, and this agree with the distribution of minerals in the thin sections.

c) The correlation matrix between $C A I$, minerals and EQC shows that the $C A I$ values with the plagioclase are logical in content and size of the crystal. Also with amphibole, but it does not have seemingly sense the variation that the clinopiroxeno presents. However, although 
the value of the correlation coefficient $(0.78)$ is low, the most significant is the negative sign. This indicates that the greater existence of clinopyroxene lower $C A I$.

d) A strong linear correlation was found between $C A I$ and $\mathrm{SiO}_{2}\left(R^{2}\right.$ equal to $\left.88.3 \%\right)$, as well as between $C A I$ and plagioclase grain size ( $R^{2}$ equal to $\left.87.5 \%\right)$.

e) A no clear relation was found between $C A I$ and $E Q C$ (Equivalent Quartz Content).

f) Relation between $C A I$ and plagioclase content was found not to be strong ( $R^{2}$ equal to $68.8 \%$ ). Similarly, correlation between $C A I$ and $\mathrm{Al}_{2} \mathrm{O}_{3}$ or $\mathrm{Fe}_{2} \mathrm{O}_{3}$ was also rather weak $\left(R^{2}\right.$ equal to $66.8 \%$ and $48.1 \%$, respectively). Especially, it is interesting to mention that the two oxides were found not to be statistically significant when performing a multiregression analysis between $C A I$ and chemical compounds.

g) An estimation of $C A I$ for the andesitic rocks of central Ecuador may be performed using the linear regressions obtained in this paper for plagioclase grain size and/or the content in $\mathrm{SiO}_{2}$, $\mathrm{FeO}, \mathrm{MgO}, \mathrm{CaO}, \mathrm{Na}_{2} \mathrm{O}$ and $\mathrm{K}_{2} \mathrm{O}$ compounds (multiregression). The use of those relations will enable an easy and fast estimation of $C A I$ without the necessity of performing any Cerchar abrasivity test.

h) Comparison of $C A I$ vs. the modal compounds of the plagioclase and $C A I$ vs. the rest of the compounds shows that while plagioclase results in a clear positive influence on $C A I$, the content of the other compounds tend to lower the index.

\section{Acknowledgements}

This research did not receive any specific grant from funding agencies in the public, commercial, or not-for-profit sectors.

\section{References}

NF P 94-430-1, 2000. Determination du pouvoir abrasif d'une roche- Partie 1: Essai de rayure avec une pointe. Association française de Normalisation (AFNOR), Paris. 
Al-Ameen, S.L., Waller, M.D., 1994. The influence of rock strength and abrasive mineral content on the CERCHAR abrasive index. Eng. Geol. 36, 293-301.

Alber, M., 2007. Stress dependency of the Cerchar Abrasivity Index (CAI) and its effects on wear of selected rock cutting tools. Tunn. Undergr. Space Technol. 9, 351-539.

Alber, M., 2008. Stress dependency of the Cerchar abrasivity index (CAI) and its effects on wear of selected rock cutting tools. Tunn. Undergr. Space Technol. 23, 351-359.

Alber, M., Yaralı, O., Dahl, F., Bruland, A., Käsling, H., Michalakopoulos, T.N., Cardu, M., Hagan, P., Aydın, H, Özarslan, A., 2014. ISRM suggested method for determining the abrasivity of rock by the CERCHAR abrasivity test. Rock Mech. Rock Eng. 47, 261-266.

ASTM D3967, 2001. Standard test method for splitting tensile strength of intact rock core specimens. American Society for Testing and Materials, West Conshohocken, PA.

ASTM D7012, 2010. Standard test method for compressive strength and elastic module of intact rock core specimens under varying states of stress and temperatures. American Society for Testing and Materials, West Conshohocken, PA.

ASTM D7625, 2010. Standard Test Method for Laboratory Determination of Abrasiveness of Rock Using the CERCHAR Method. American Society for Testing and Materials, West Conshohocken, PA.

Atkinson, T., Cassapi, V.B., Singh, R.N., 1986a. Assessment of abrasive wear resistance potential in rock excavation machinery. Int. J. Min. Geol. Eng. 3, 151-163.

Atkinson, T., Denby, B., Cassapi, V.B., 1986b. Problems associated with rock material properties in surface mining equipment selection. Trans. Inst. Min. Metall. Section. A Miner. Ind. 95, A80-A86.

Boland, M.P., Pilatasig, L.F., Ibandango, C.E., McCourt, W.J., Aspden, J.A., Hughes, R.A., Beate B., 2000. Geology of the Western Cordillera between $0^{\circ}-1^{\circ} \mathrm{N}$, Mining Development and 
Environmental Control Project, Map and Geological Information Program, Report No. 10,

(Proyecto de Desarrollo Minero y Control Ambiental, Programa de Informacion cartografica y Geológica, Informe No. 10), CODIGEM-BGS, Quito, Ecuador, pp 72. In Spanish.

CERCHAR, 1986. The CERCHAR abrasiveness index. Centre d'Etudes et des Recherches des Charbonages de France, Verneuil, France.

Deliormanl1, A., 2011. Cerchar abrasivitiy index (CAI) and its relation to strength and abrasion test methods for marble stones. Constr. Building Mat. 30, 16-21.

Deliormanl1, A.H., 2012. Cerchar abrasivity index (CAI) and its relation to strength and abrasion test methods for marble stones. Constr. Build Mater. 30, 16-21.

Er, S., Tugrul, A., 2016a. Correlation of physico-mechanical properties of granitic rocks with Cerchar Abrasivity Index in Turkey. Measurement 91, 114-123.

Er, S., Tugrul, A., 2016b. Estimation of Cerchar abrasivity index of granitic rocks in Turkey by geological properties using regression analysis. B. Eng. Geol. Environ. 75(3), 1325-1339.

Fowell, R.J., Abu Bakar, M.Z., 2007. A review of the Cerchar and LCPC rock abrasivity measurement methods. Proceeding of the 11th Congress of the International Society for Rock Mechanics 155-160.

Hamzaban, M.T., Memarian, H., Rostami, J., 2014. Continuous monitoring of pin tip wear and penetration into rock surface using a new Cerchar abrasivity testing device. Rock Mech. Rock Eng. 47(2), 689-701.

Hamzaban, M.T., Memarian, H., Rostami, J., Ghasemi-Monfared, H., 2014. Study of rock-pin interaction in Cerchar abrasivity test. Int. J. Rock Mech. Min. Sci. 72, 100-108.

ISRM, 2007. The Complete ISRM suggested methods for rock characterization, testing and monitoring: 1974-2006. International Society for Rock Mechanics, Lisbon. 
Kahraman, S., Alber, M., Fener, M., Gunaydin, O., 2010. The usability of Cerchar abrasivity index 388 for the prediction of UCS and E of Misis Fault Breccia: regression and artificial neural networks analysis. Expert Syst. Appl. 37, 8750-8756.

Käsling, H., Thuro, K., 2010. Determining abrasivity of rock in the laboratory. European Rock Mechanics Symposium. EUROCK 2010, Laussane, Switzerland.

Lassnig, K., Latal, C., Klima, K., 2008. Impact of grain size on the Cerchar abrasiveness test. Ernst 393 and Sohn Verlag für Architektur und technische Wissenschaften $\mathrm{GmbH}$ and Co. KG. Berlin 394 Geomechanik und Tunnelbau 1, Heft 1.

Majeed. Y., Abu Bakar, M.Z., 2016. Statistical evaluation of CERCHAR Abrasivity Index (CAI) 396 measurement methods and dependence on petrographic and mechanical properties of selected rocks 397 of Pakistan. Bull. Eng. Geol. Environ. 75, 1341-1360.

Michalakopoulos, T.N., Anagnostou, V.G., Bassanou, M.E., Panagiotou, G.N., 2005. The influence 399 of steel styli hardness on the Cerchar abrasiveness index value. Inter. J. Rock Mech. Mining. Sci. 400 Geomechan. Abstracts 43, 321-327. properties for predicting cerchar abrasiveness index (CAI) in sandstones. Int. J. Emerg. Technol. Advan. Eng. 3(9), 99-109.

Plinninger, R., Kasling, H., Thuro, K., Spaun, G., 2003. Testing conditions and geomechanical 405 properties in influencing the CERCHAR abrasiveness index (CAI) value. J. Rock Mech. Mining 406 Sci. 40, 159-263. factors affecting cerchar abrasivity index. Mech. Rock Eng. 47, 1905-1919. 
StatPoint Technologies, Inc., 2009. STATGRAPHICS Centurion XVI User Manual. The Plains, VA.

Suana, M., Peters, T., 1982. The CERCHAR abrasivity index and its relation to rock mineralogy and petrography. Rock Mech. Rock Eng. 15, 1-7.

Thuro, K., 1997. Prediction of drillability in hard rock tunneling by drilling and blasting, in: Golser, Hinkel and Schubert (Eds.), Tunnels for People, Balkema, Rotterdam, pp. 103-108.

Vallejo, C., 2007. Evolution of the Western Cordillera in the Andes of Ecuador (Late CretaceousPaleogene). PhD. Dissertation, Institute of Geology, ETH Zürich.

Vallejo, C., Winkler, W., Spikings, R.A., Luzieux, L., Heller, F., Bussy, F., 2009. Mode and timing of terrane accretion in the forearc of the Andes in Ecuador, in: Kay, S.M., Ramos, V.A., Dickinson, W.R. (Eds.), Backbone of the Americas: Shallow Subduction, Plateau Uplift, and Ridge and Terrane Collision, Geol. Soc. Am. Mem. 204.

Vera, R.H., 2016. Geology of Ecuador. Iberia, Quito.

Vezzoli, L., Apuani, T., Corazzato, C., Uttini, A., 2017. Geological and geotechnical characterization of the debris avalanche and pyroclastic deposits of Cotopaxi Volcano (Ecuador). A contribute to instability-related hazard studies. J. Volcanol. Geotherm. Res. 332, 51-70.

West, G., 1989. Rock abrasiveness testing for tunneling. Int. J. Rock Mech. Min. Sci. Geomech. Abstr. 26, 151-160.

Yarali, O., Yasar, E., Bacak, G., Ranjith, P.G., 2008. A study of rock abrasivity and tool wear in coal measures rocks. Int. J. Coal Geol. 74, 53-66. 